PROSTATE CANCER

\section{Mutations in ctDNA reflect features of metastatic disease}

Regular biopsy sampling enables the personalized management of patients with metastatic castration-resistant prostate cancer (mCRPC); however, such sampling is invasive, carries the risk of complications, and might fail to capture all of the specific characteristics of a patient's disease. Now, a systematic comparison of matched solid tumour and liquid biopsy samples confirms the feasibility of relying entirely on information provided by liquid, rather than solid, biopsy sampling.

Investigators obtained 45 matched tumour and liquid biopsy samples from patients with $\mathrm{mCRPC}$. The presence of somatic mutations in a total of 72 clinically relevant genes was then investigated using targeted sequencing. Circulating tumour (ct)DNA levels exceeded $2 \%$ of total cell-free (cf)DNA in 34 liquid biopsy samples (75.6\%); however, the cfDNA fraction was not measurable in five samples, owing to a lack of detectable mutations. AR amplifications were detected in 22 ctDNA samples (64.7\%), with SPOP mutations detected in three. Inactivating mutations in several tumour suppressors, including TP53, $B R C A 2, A P C$, and others were also detected. A total of 78 somatic mutations were detected in tumour biopsy samples from patients with detectable levels of ctDNA; remarkably, 73 of these (93.6\%) were also identified in ctDNA. Analysis of ctDNA samples revealed a further 36 somatic mutations that were not detected in biopsy samples. The authors attributed the majority of these undetected mutations to insufficient sequencing coverage; however, seven mutations, including possible driver mutations in TP53, RB1 and PTEN were detected in ctDNA, but not in the matched tumour biopsy sample.

These data demonstrate the potential for liquid biopsy sampling to replace traditional biopsy sampling approaches, although prospective confirmation of noninferior patient outcomes will likely also be required.

Peter Sidaway

This article is modified from the original in Nat. Rev. Clin. Oncol. (http://dx.doi.org/10.1038/nrclinonc.2017.111).

ORIGINAL ARTICLE Wyatt, A. W. et al. Concordance of circulating tumor DNA and matched metastatic tissue biopsy in prostate cancer.J. Natl Cancer Inst. http://dx.doi. org/10.1093/jnci/djx118 (2017) 